\title{
Analysis of Lift and Drag of Mono-foil Hysucat due to Longitudinal Foil-placement Variation
}

\author{
Ketut Suastika $^{1}$, Regi Y. Dikantoro ${ }^{1}$, Dedi B. Purwanto ${ }^{1}$, Dony Setyawan ${ }^{1}$, Wing H. A. Putra ${ }^{1}$
}

\begin{abstract}
CFD simulations were conducted to study the effects of a hydrofoil and its relative placement in the longitudinal direction on the total resistance of a mono-foil hysucat (hydrofoil supported catamaran). Three foil positions were considered: (i) precisely below the vessel's center of gravity, (ii) 3 chord-lengths aft from position 1 and (iii) 6 chordlengths aft from position 1. At relatively low speed (volumetric Froude number FnV $<1.8$ ), the hydrofoil results in an increase of the total resistance of the hysucat (up to $4.43 \%)$. At relatively high speed $(\mathrm{FnV}>1.8)$, the hydrofoil results in a decrease of the total resistance (up to $34.86 \%$ ). The resistance coefficient first increases, takes a maximum value and then decreases with increasing Froude number. The maximum value is observed at $\mathrm{FnV} \approx 1.4$ (or Fn $\approx 0.5)$, consistent with previous observations. The most optimum foil placement is that precisely below the center of gravity of the vessel.
\end{abstract}

Keywords-Catamaran, CFD, Hydrofoil, hysucat, Resistance.

\section{INTRODUCTION}

$\mathrm{H}$ ydrofoil supported catamaran (hysucat) is a hybrid vessel that combines the use of one or more hydrofoils on a catamaran. This type of vessel has a greater efficiency than a fast boat of catamaran type [1-3]. The application of a hydrofoil results in a decrease of the draft, which results in a decrease of frictional resistance and wavemaking resistance [4]. Furthermore, Miyata [5] also reported that the use of hydrofoils on a catamaran improves the resistance and seakeeping characteristics.

Manoharan and Sahoo [6] developed an analytical method to calculate the drag of NPL round bilge hull forms in hysucat configurations. They found that increasing the foil's span with a fixed chord-length improves resistance characteristics. On the contrary, changing the chord-length with fixed span does not affect the resistance characteristics to a large extent. The improvement in resistance characteristics with foil moving forward was due to the planing effect caused by the increased stern trim obtained. Considerable resistance improvement has been achieved with hydrofoil assistance for Froude numbers of 0.8 and above. The improved wave resistance characteristics in low Froude numbers were offset by the added frictional resistance. The results of numerical simulation indicate that substantial reduction in drag can be achieved. However, experimental tests need to be performed to validate the analytical solution.

Andrewartha et al. [7] presented a numerical method to calculate the resistance of foil-assisted catamaran for varying design parameters (number of foil, foil size, longitudinal and vertical position as well as load condition), allowing a wide range of parameters to be tested in the preleminary design of foil-assisted catamaran. Their method is capable of predicting the resistance of a foil-assisted catamaran within reasonable accuracy. It was shown that a significant interaction occurs between the foil and the hull; the frictional resistance of the demihulls is increased due to the surface wave produced by the foil.

The purpose of the present study is to investigate the effects of an application of a hydrofoil (mono foil) on the total resistance of a hysucat. For that purpose, computational fluid dynamic (CFD) simulations were performed. A round bilge symmetrical catamaran is considered, for which the main particulars are summarised in Table 1. Figure 1 shows the body plan of a demi hull and Fig. 2 shows the general arrangement of the catamaran [8]. In the present study, a hydrofoil system is retrofitted to an existing catamaran design to improve its efficiency.

Four cases were considered in the simulations of the catamaran with and without foil whereby three variations of foil placement in the longitudinal direction were studied, as summarised in Table 2 . The submerged depth of the foil is $h=0.4 \mathrm{~m}$ below the free surface $(h / c=0.5$, where $c$ is the chord length), which is $20 \%$ deeper than the maximum recommended submerged depth by Hoppe [9] to ensure that the foil still below the water level at relatively high speed $(0.15<h / c<0.4)$. 
International Journal of Marine Engineering Innovation and Research, Vol. 2(2), Mar. 2018. 176-181

Tabel 1. Main particulars of the catamaran.

\begin{tabular}{lr}
\hline $\mathrm{L}_{\mathrm{WL}}$ (Length at water line) & $44.0 \mathrm{~m}$ \\
$\mathrm{~B}$ (breadth) & $11.8 \mathrm{~m}$ \\
$\mathrm{~T}$ (Draft) & $1.4 \mathrm{~m}$ \\
$\mathrm{H}$ (Height) & $3.8 \mathrm{~m}$ \\
$\mathrm{~V}_{\mathrm{s}}$ (Service speed) & $28.0 \mathrm{kn}$ \\
$\mathrm{B}_{1}$ (Breadth of the demi hull) & $3.0 \mathrm{~m}$ \\
Distance between demi hulls & $5.8 \mathrm{~m}$ \\
Displacement (without foil) & $185.2 \mathrm{t}$ \\
Displacement with foil & $186.5 \mathrm{t}$ \\
\hline
\end{tabular}

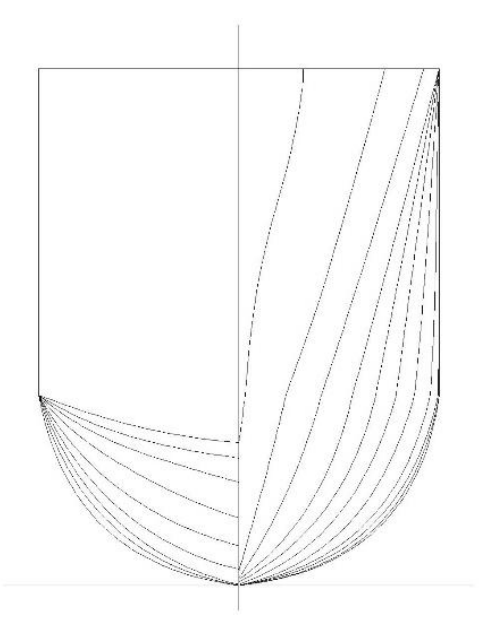

Figure. 1. Body plan of a demi hull [8].

Table 2. Simulation cases.

\begin{tabular}{cc}
\hline Simulation's name & Description \\
\hline Bare hull & Without foil \\
Foil position 1 & Foil below the center of gravity CG \\
Foil position 2 & Foil 3-c aft position 1 $(c=$ chord length $)$ \\
Foil position 3 & Foil $6-c$ aft position $1(c=$ chord length $)$ \\
\hline
\end{tabular}



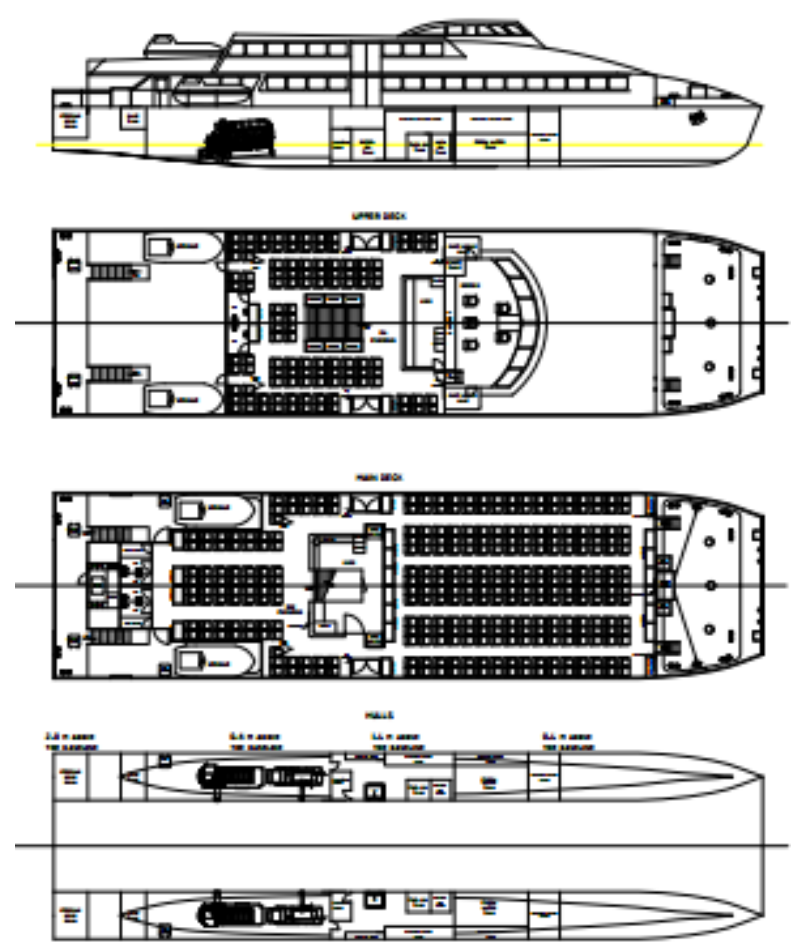

Figure. 2. General arrangement of the catamaran [8].

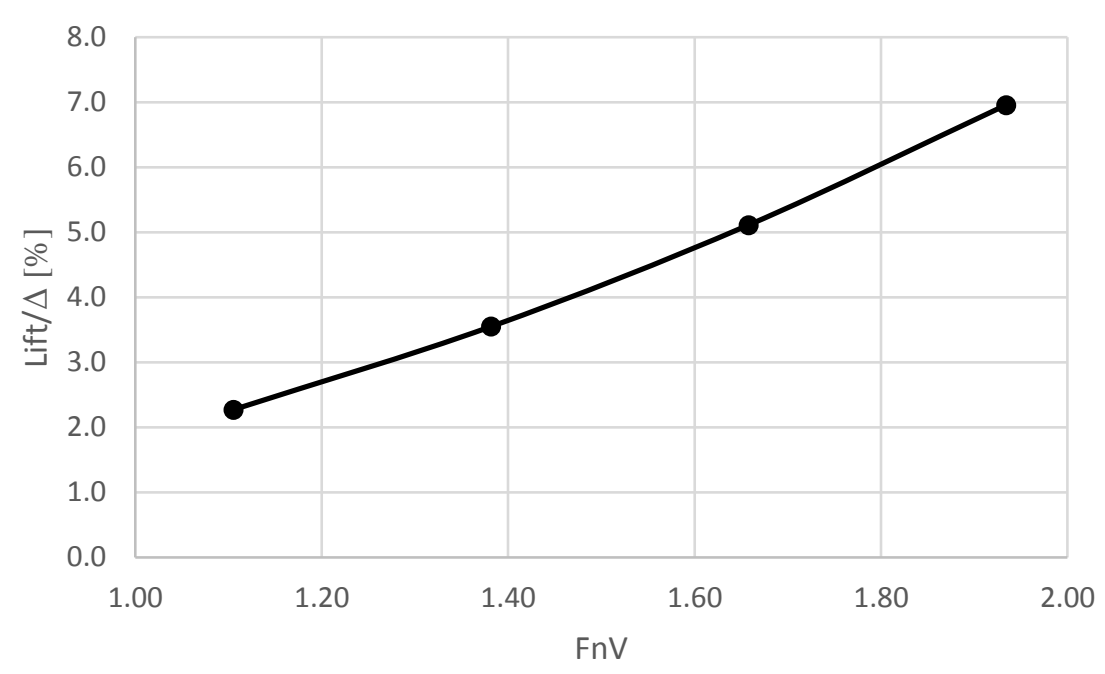

Figure 3. Foil lift as function of volumetric Froude number (simulations of foil alone).

\section{METHOD}

\section{A. Simulations of Foil Alone}

CFD simulations of foil alone were carried out to determine the most optimum foil size [10]. The foil used was NACA 64(1)212. The optimum angle of attack was found to be 2 degrees. The strut was NACA 0010 symmetrical foil. The foil was designed to support the catamaran by generating a lift approximately $7 \%$ of the catamaran displacement at the service speed $\left(\mathrm{Vs}_{\mathrm{s}}=28\right.$ knots; FnV = 1.93; see Fig. 3). Simulation results show that the optimum foil size has a chord length of $0.8 \mathrm{~m}$ and a span of $5.2 \mathrm{~m}$ (aspect ratio $=6.5)$.

\section{B. Simulations of Catamaran with and without Foil}

The Fine/Marine ${ }^{\circledR}$ software from Numeca was used for the simulations of catamaran with and without foil. The computational domain is defined with the inlet $1.0 \mathrm{~L}$ upstream from the vessel, the outlet $3.0 \mathrm{~L}$ behind the vessel and the side walls $1.5 \mathrm{~L}$ aside the vessel, where $L$ is the length between the perpendiculars. Furthermore, the bootom wall is located $1.50 \mathrm{~L}$ below the vessel and the top wall is $0.5 L$ above the vessel. Free surface effects (generaion of waves) are modeled in the simulations. The boundary conditions and the simulation parameters are summarised in Table 3.

Grid-independence tests were carried out to ensure that the numerical results (resistance, lift) do not depend on the number of elements (cells) being used in the simulations. Figure 4 shows a mesh of a demi hull with foil and strut for the case where the foil is located precisely below the center of gravity (foil position 1). The number of elements 
International Journal of Marine Engineering Innovation and Research, Vol. 2(2), Mar. 2018. 176-181 (pISSN: 2541-5972, eISSN: 2548-1479

used in the optimum setting was two million and hundred thousands $\left(2.1 \times 10^{6}\right)$.

Table 3. Simulation parameters.

\begin{tabular}{|c|c|c|}
\hline Description & Type & Condition \\
\hline Xmin (Inlet) & EXT & Far field, $\mathrm{Vx}=0$ \\
\hline Xmax (Outlet) & EXT & Far field, $V x=0$ \\
\hline Zmin (Bottom) & EXT & Updated Hydrostatic Pressure \\
\hline Zmax (Top) & EXT & Updated Hydrostatic Pressure \\
\hline Ymin (Side) & MIR & Mirror \\
\hline Ymax(side) & EXT & Far field, $V x=0$ \\
\hline Ship and foil & SOL & Wall Function \\
\hline Motion & $\begin{array}{l}\text { Translation in } \mathrm{X} \text { direction with } \\
\text { a given speed }\end{array}$ & $\begin{array}{l}\text { Speed = ship speed (for example, } \\
28 \text { knots) using } 1 / 2 \text { sinusoidal ramp }\end{array}$ \\
\hline $\begin{array}{l}\text { Convergence } \\
\text { criterion }\end{array}$ & $\begin{array}{l}\text { Number of order of magnitude } \\
\text { the residual must decrease }\end{array}$ & 2 (Second order) \\
\hline
\end{tabular}

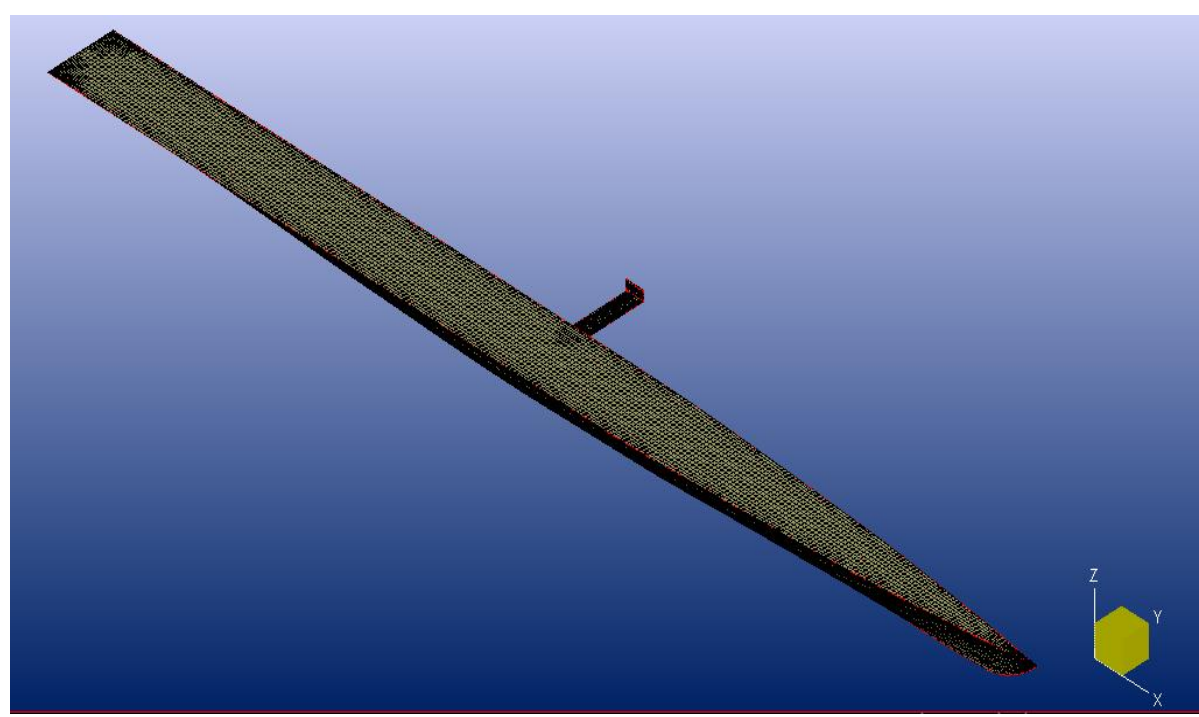

Figure 4. Mesh of a half of the catamaran (a demi hull, a half-span foil and a half strut). 


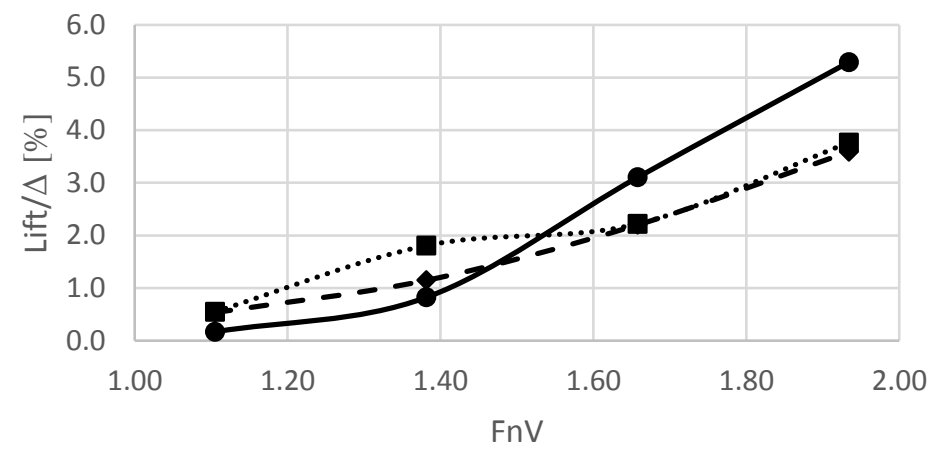

$\longrightarrow$ Foil position $1-\bullet-$ Foil Position $2 \cdots \cdots$ Foil Position 3

Figure 5. Foil lift as function of volumetric Froude number (simulations of foil attached to the catamaran).

\section{RESULTS AND DISCUSSION}

Figure 5 shows the foil lift as function of volumetric Froude number for three different foil placements in the longitudinal direction. For all foil positions, the lift force increases with increasing Froude number, as expected. A comparison to Fig. 3 shows that the demi hulls influence the magnitude of the foil lift, that is, they decrease the foil lift (a decrease that can reach a value of more than $50 \%$ in the lower Froude numbers depending on the foil relative placement in the longitudinal direction). This observation is consistent with that reported previously by Ishikawa [11]. Figure 5 also shows that the magnitude of the lift force at a given speed (a particular Froude-number) depends on the foil relative placement in the longitudinal direction. At relatively high speed $(\mathrm{FnV}>1.6)$, the foil placement 1 (below the center of gravity) gives the largest foil lift.

Figure 6 shows the total ship resistance (fraction of displacement) as function of volumetric Froude number. Figure 6 shows that, at relatively low speed $(1.4<\mathrm{FnV}<$ 1.75), the hydrofoil results in an increase of the total resistance (up to $4.43 \%$ ). This is ascribed to the drag of the foil (and the strut) while the lift has not been built sufficiently to lift the hull (reducing the wetted surface area). At relatively high speed $(\mathrm{FnV}>1.8)$, the hydrofoil results in a decrease of the total resistance (up to $34.86 \%$ ). The largest decrease is obtained for foil placement 1 (below the center of gravity). This observation is consistent with the simulation results of foil lift, for which the foil placement 1 gives the largest lift. Migeotte [12] reported that the effect of decreasing resistance due to a foil was observed at FnV > 2.0, which is in a good agreement with the present finding.

Figure 7 shows the resistance coefficient $C_{T}$ as function of volumetric Froude number. The resistance coefficient is calculated as

$$
C_{T}=\frac{R_{T}}{0.5 \rho S V^{2}}
$$

where $R_{T}$ is the total resistance, $\rho$ is the mass density of sea water $\left(1025 \mathrm{~kg} / \mathrm{m}^{3}\right), S$ is the wetted surface area and $V$ is the ship's speed. The resistance coefficient first increases, takes a maximum value and then decreases with increasing Froude number. The maximum value takes place at $\mathrm{FnV} \approx 1.4$ (or $\mathrm{Fn} \approx 0.5$ ). This observation is consistent with those reported in $[10,13,14]$.

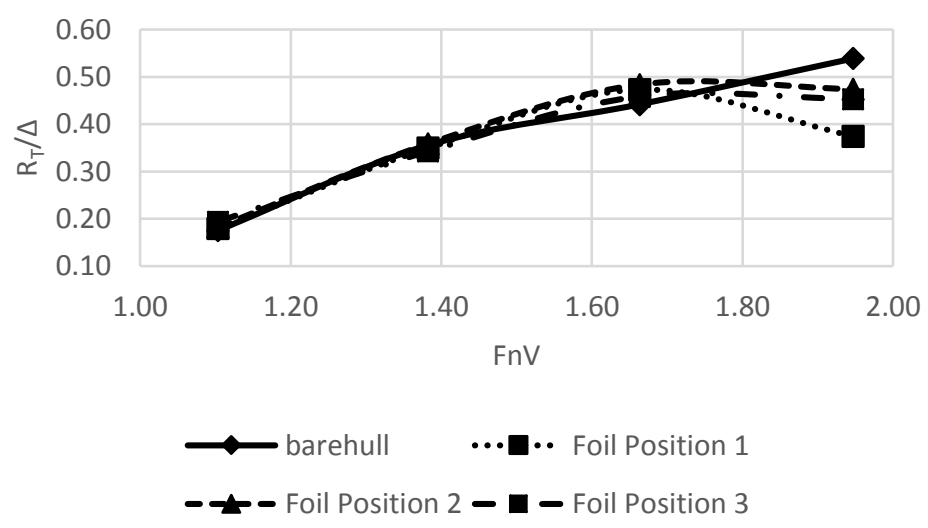

Figure 6. Total ship resistance (fraction of displacement) as function of volumetric Froude number. 


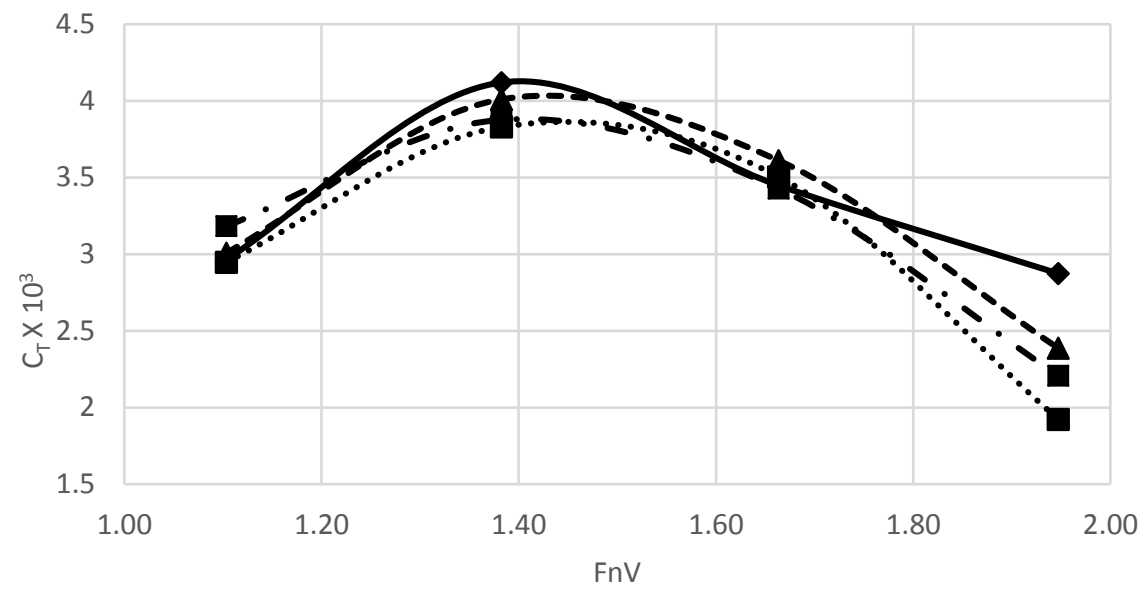

$\neg$ barehull … $\square$... Foil Position $1-\boldsymbol{- t}-$ Foil Position $2-\mathbf{a}-$ Foil Position 3

Figure 7. Resistance coefficient as function of volumetric Froude number.

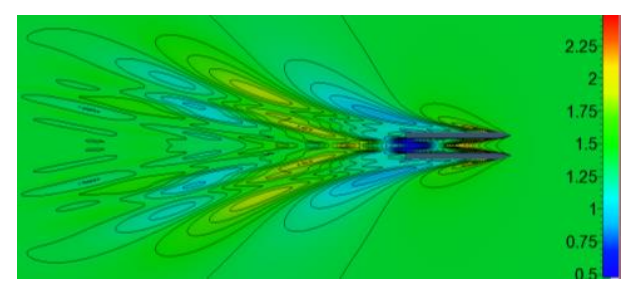

(a) Bare hull

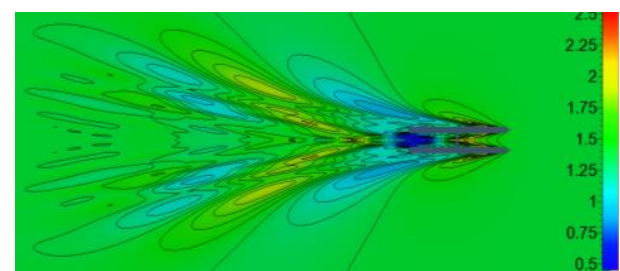

(b) Foil position 1

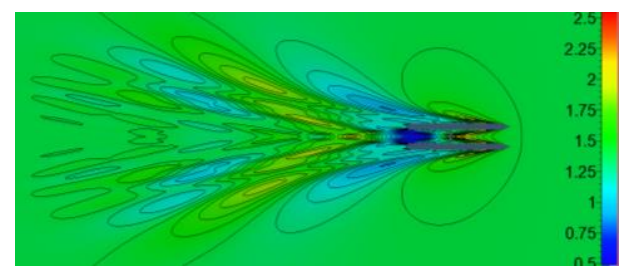

(c) Foil position 2

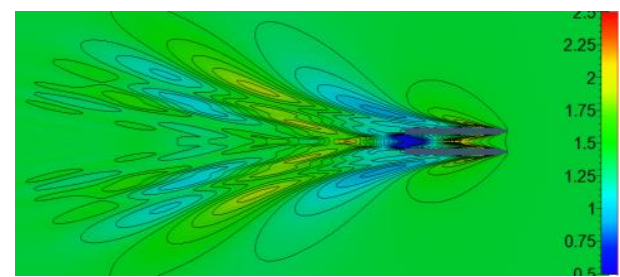

(d) Foil position 3

Figure 8. Wave pattern for bare hull (a), foil position 1 (b), foil position 2 (c) and foil position 3 (d); Vessel's speed $=28$ knots $(\mathrm{FnV}=1.93)$. 
Figure 8 shows the wave pattern for ship speed of 28.0 knots $(\mathrm{FnV}=1.93)$. As shown in Fig. 8, the foil does not change the wave pattern significantly regardless the foil relative placement in the longitudinal direction. This is contrary to the prediction made by the model of Andrewartha et al. [7], in which the foil produces surface waves significantly to increase the frictional resistance of the demi hulls. Experiments are required to verify the present numerical results and the model prediction.

\section{CONCLUSION}

CFD simulations were conducted to study the effects of a hydrofoil and its relative placement in the longitudinal direction on the total resistance of a mono-foil hysucat. Three foil positions were considered: (i) precisely below the vessel's center of gravity, (ii) 3 chord-lengths aft from position 1 and (iii) 6 chord-lengths aft from position 1 . Simulations of foil alone and of a catamaran with and without foil show that the demi hulls and the foil relative placement in the longitudinal direction significantly affect the foil lift and the hysucat's total resistance. Generally, the demi hulls decrease the foil lift as compared to that of foil alone (without demi hulls). This observation is consistent with that reported earlier by Ishikawa [11]. At relatively low speed $(\mathrm{FnV}<1.8)$, the hydrofoil results in an increase of the total resistance of the hysucat (up to $4.43 \%)$. On the contrary, at relatively high speed ( $\mathrm{FnV}>$ 1.8 ), the hydrofoil results in a decrease of the total resistance (up to $34.86 \%$ ). Experiments are required to verify the simulation results.

The resistance coefficient first increases, takes a maximum value and then decreases with increasing Froude number. The maximum value is observed at FnV $\approx 1.4$ (or Fn $\approx 0.5$ ). This observation is consistent with those previously reported in $[10,13,14]$. From the simulations considered, the most optimum placement for the hydrofoil is that precisely below the center of gravity of the vessel.

\section{ACKNOWLEDGEMENT}

This research project was financially supported by the LPPM ITS Surabaya under the grant Penelitian Dana Departemen with contract no.1210/PKS/ITS/2017.

\section{REFERENCES}

[1] K. Hoppe, “The hysucat development," Dept. Mech. Eng., Univ. Stellenbosch, South Africa, 1989.

[2] K. Hoppe, "Optimisation of hydrofoil-supported planing catamarans," Fast Sea Transportation, 1995.

[3] G. S. Milandri, "Seakeeping control of HYSUCATs," M.S. thesis, Univ. Stellenbosch, South Africa, 2006.

[4] K. G. Hoppe, "Recent applications of hydrofoil-supportedcatamarans," Fast Ferry International, pp. 1-20, 2001.

[5] H. Miyata, "Development of a new-type hydrofoil catamaran," $J$. Ship Res., vol. 33, no. 2, pp. 135-144, 1989.

[6] R. Manoharan and P. K. Sahoo, "Drag reduction of NPL round bilge hull forms in HYSUCAT configuration: An analytical study," $7^{\text {th }}$ Int. Conf. High-Performance Marine Vehicles, Melbourne, Florida, USA, 13-15 October 2010, pp. 235-251, 2010.

[7] M. Andrewartha, L. Doctors, K. Kantimahanthi, and P. Brandner, "A numerical method for performance prediction of hydrofoil assisted catamarans," Proc. $7^{\text {th }}$ Int. Conf. Fast Sea Transportation (FAST '03), Naples, Italy, pp 9-16., 2003.

[8] M. H. Satriawansyah, "Design of catamaran passenger ship for the route of Boom Marina, Banyuwangi to Benoa Port, Final Project Rep., Dept. Naval Arch., Fac. Marine Tech., ITS Surabaya, Indonesia, 2016.

[9] K. G. Hoppe, U.S. Patent 4606 291, Aug 19, 1986.

[10]K. Suastika, A. Hidayat, S. Riyadi, "Effects of the application of a stern foil on ship resistance: A case study of an Orela crew boat,' Int. J. Tech., vol. 8, no. 7, pp. 1266-1275, 2017.

[11]S. Ishikawa, "Study on hydrodynamic interaction between hull and submerged foils," Spring Meeting SNAME, 1991.

[12] G. Migeotte, "Hydrofoil supported catamaran," Professional Boat Builder, no. 157, Boston Boatworks, 2015.

[13]R. Marshall, Powering Your Boat. All About Powerboats: Understanding Design and Performance, McGraw-Hill Prof., New York, 2002.

[14]R. Yousefi, R. Shafaghat, M. Shakeri, "Hydrodynamic analysis techniques for high-speed planing hulls," Applied Ocean Res., vol. 42, pp. 105-113, 2013. 